\title{
Cryptococcus Neoformans: Primeiro Caso \\ Relatado de Isolamento em Fezes de Pombos no \\ Município de Votuporanga-São Paulo
}

Cátia Rezende*

Eliani Rodrigues da Silva**

Alexandre Henrique Frota Gomes ***

João Edson da Silva***

Renata Camacho Mizziara****

\section{RESUMO}

Cryptococcus neoformans e Cryptococcus gattii são fungos patogênicos causadores de pneumonia e meningite em pacientes imunodebilitados e imunocompetentes. Ambas as espécies são encontradas no meio ambiente e adquiridas via inalação, ocasionando uma infecção pulmonar inicial. Foram avaliadas 48 amostras de ar e 32 amostras de fezes de pombos entre junho e agosto de 2007. A presença de cápsula foi examinada pela microscopia com Tinta Nanquim. As amostras também foram testadas para produção de urease e da enzima fenoloxidase. Cryptococcus neoformans foi isolado em $3,1 \%$ de fezes de pombos. Os resultados sugerem que condições climáticas podem afetar a ocorrência da levedura em diferentes fontes ambientais.

Palavras-Chave: Fezes de pombos; Ar; Cryptococcus neoformans; Votuporanga.

\section{INTRODUÇão}

O Cryptococcus neoformans é um fungo patogênico oportunista causador de

\footnotetext{
*Mestre em Biotecnologia (Instituto Química-UNESP), professora-titular de Microbiologia do curso de Farmácia e Biomedicina do Centro Universitário de Votuporanga.

**Doutora em Ciências Biológicas (IBILCE-UNESP), professora do curso de Ciências Biológicas do Centro Universitário de Votuporanga.

***Biólogos formados no Centro Universitário de Votuporanga.

****Doutora em Genética (USP), professora-assistente de Microbiologia do curso de Farmácia do Centro Universitário da FEB.

*Autor-correspondente: Cátia Rezende.

Endereço: Rua Pernambuco, 4.196 - Centro - CEP: 15.500-006 - Votuporanga-SP.

E-mail: catia_rezende@terra.com.br.
} 
pneumonia e meningite, de distribuição mundial (FILIÚ et al., 2002; QUINTERO; CASTAÑEDA; RUIZ, 2005). As variedades neoformans e grubii são responsáveis pela infecção em pacientes imunodebilitados. Já o Cryptococcus gatti infecta, preferencialmente, pacientes imunocompetentes (QUINTERO; CASTAÑEDA; RUIZ, 2005; PUKKILA-WORLEY; MYLONAKIS, 2008;VELAGAPUDI et al., 2009). Ambas as espécies são encontradas no meio ambiente e adquiridas pela inalação de propágulos infectantes que causam infecção pulmonar primária (VELAGAPUDI et al., 2009; CASTAÑEDA; RUIZ, 2005). Há hipóteses de que os propágulos infectantes sejam pequenas células leveduriformes dessecadas ou esporos produzidos pela reprodução sexual da levedura no ambiente (VELAGAPUDI et al., 2009).

O habitat natural do Cryptococcus gatti no meio ambiente foi demonstrado por pesquisadores australianos por meio da associação com espécies de eucaliptos (CALLEJAS et al., 1998; KROCKENBERGER; CANFIELD; MALIK, 2002; CAMPISI et al., 2003; DAVEL et al., 2003; GUGNANI et al., 2005; QUINTERO; CASTAÑEDA; RUIZ, 2005). Estudos desenvolvidos em várias regiões do mundo têm demonstrado a presença do $C$. gatti em diversas espécies de árvores em regiões tropicais e subtropicais (GROVER et al., 2009; GRANADOS; CASTAÑEDA, 2006; ESCANDÓN et al., 2005; DAVEL et al., 2003; KROCKENBERGER; CANFIELD; MALIK, 2002; FORTES et al., 2001; CALLEJAS et al., 1998).

Os reservatórios de C. neoformans variedade neooformans e grubii são pombos e pássaros domésticos frequentemente saudáveis, que eliminam em seus excrementos a levedura, contribuindo com a propagação na natureza (PAL, 1997; QUINTERO; CASTAÑEDA; RUIZ, 2005; SOOGARUN et al., 2006). Entretanto, vários estudos têm demonstrado a presença dessa espécie em folhas em decomposição de diferentes espécies de árvores e em sujeiras domésticas (GROVER et al., 2009; GRANADOS; CASTAÑEDA, 2006; GUGNANI et al., 2005; QUINTERO; CASTAÑEDA; RUIZ, 2005; ERGIN et al., 2004; CAMPISI et al., 2003).

Baltazar e Ribeiro (2006) isolaram a variedade $C$. gattii e $C$. neoformans de amostras de árvores em diferentes regiões do Estado do Espírito Santo. Outro estudo realizado em Campo Grande, Mato Grosso do Sul, demonstrou a presença de $C$. neoformans em fezes de pombos (FILIÚ et al., 2002). Passoni e colaboradores (1998) associaram C. neoformans em fezes de pombos e poeiras domésticas na cidade do Rio de Janeiro.

A investigação de surtos comunitários de criptococose tem contribuído numa melhor compreensão de fontes de transmissão (ELLIS et al., 2000), incentivando os estudos que possam identificar o nicho ecológico dessa levedura.

Compreender a ecologia do C. neoformans e do C. gattii é fundamental na 
epidemiologia da criptococose, pois estabelecendo possíveis habitats se podem desenvolver medidas preventivas minimizando a transmissão. Este estudo objetiva avaliar a presença de espécies de Cryptococcus em amostra ambientais e de fezes de pombos na cidade de Votuporanga-SP.

\section{Metodologia}

Amostras de fezes de pombos e do ar foram coletadas em diferentes regiões do município de Votuporanga-SP, no período de junho a agosto de 2007. As regiões analisadas foram: central, leste, sul e norte (Figura 01). Para cada região analisada foram coletadas 12 amostras do ar e 8 amostras de fezes, totalizando 48 e 32, respectivamente.

Coleta de fezes de pombos: quantidades significativas de fezes de pombos secas foram coletadas com auxílio de espátula de madeira estéril e acondicionadas em placas de Petri estéreis. As amostras foram transportadas para o laboratório Didático de Análises Clínicas do Centro Universitário de Votuporanga para processamento.

Coleta de amostra do ar: placas de Petri contendo Agar Sabouraud com cloranfenicol $0,05 \%$ foram abertas nos mesmos locais em que foram coletadas as amostras de fezes. As placas foram abertas por 15 minutos e posteriormente transportadas ao laboratório Didático de Análises Clínicas do Centro Universitário de Votuporanga para processamento.

Identificação da levedura nas amostras coletadas: As placas provenientes do ar foram incubadas em estufa bacteriológica a $30^{\circ} \mathrm{C} \pm 1^{\circ} \mathrm{C}$ durante 7 dias. Já as fezes coletadas foram processadas da seguinte maneira: $4 \mathrm{~g}$ de fezes secas foram suspendidas em $50 \mathrm{~mL}$ de solução fisiológica $0,9 \%$ estéril. Este preparado foi agitado manualmente por 5 minutos e deixado em repouso por 30 minutos. Decorrido este tempo, $4 \mathrm{~mL}$ de sobrenadante foram coletados e acondicionados em dois tubos estéreis e centrifugados por 15 minutos a 5.000rpm. O sobrenadante foi descartado e o sedimento, cultivado em meio Sabouraud com cloranfenicol $0,05 \%$. O resíduo restante foi diluído em $2 \mathrm{~mL}$ de solução fisiológica estéril 0,9\% e homogeneizado por 5 minutos. Essa suspensão foi novamente cultivada em Sabouraud com cloranfenicol. As placas foram incubadas a $30^{\circ} \mathrm{C}$ $\pm 1^{\circ} \mathrm{C}$ durante 7 dias.

Após o período de incubação, foi realizada a análise microscópica das colônias suspeitas, investigando a presença de células leveduriformes únicas e/ou com brotamento e cápsulas com Tinta Nanquim. As amostras positivas para presença de cápsula foram cultivadas em Agar uréase, Ágar Níger e Agar Yeast Nitrogen Base (auxanograma) para identificação de Cryptococcus. 


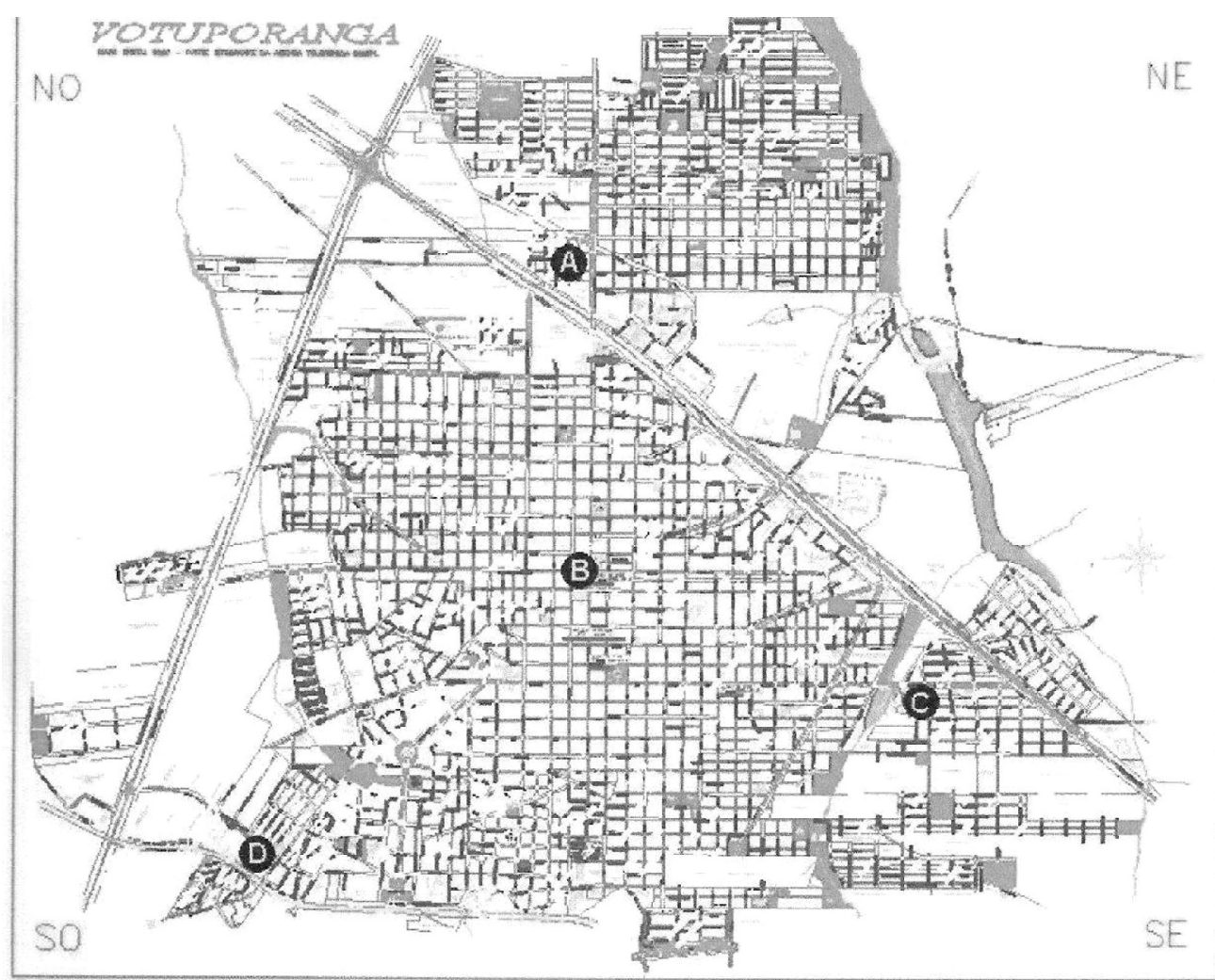

Copyright $\odot 2007$ Nelson Luiz Dumbra - http://www. dumbra.com.br

Legenda: A: Condomínio residencial - Região Norte
B: Praça Fernando Costa - Região Central
C: Clube de campo - Região Leste
D: Fábrica - Regiäo Sul

Figura 1. Mapa representativo das regiões analisadas na cidade de Votuporanga-SP.

\section{Resultados}

Ao todo, 48 amostras de ar e 32 amostras de fezes de regiões altamente povoadas por pombos no município de Votuporanga-SP foram analisadas. Das amostras de ar, apenas 01 colônia apresentou característica suspeita de Cryptococcus e, das amostras de fezes, 14 apresentaram mesma característica.

Após as provas bioquímicas para identificação de Cryptococcus neoformans, 100\% (48) das amostras do ar apresentaram ausência de Cryptococcus neoformans - e somente uma era Cryptococcus sp. Já das 32 amostras de fezes, 14 eram suspeitas de Cryptococcu sp; destas, 9 eram Cryptococcus sp e somente 1 era 
Cryptococcus neoformans (Tabela 01). Assim, das 80 amostras ambientais (48/ar e 32/fezes), somente 1,25\% era positivo para Cryptococcus neoformans ( 1 amostra/ fezes). Essa amostra foi coletada na região central (local: B) (Figura 01), sendo o local que apresentava maior índice populacional de pombos quando comparado às outras áreas analisadas.

Tabela 1. Distribuição de Cryptococcus sp e Cryptococcus neoformans isolados nas amostras de ar e fezes no município de Votuporanga.

\begin{tabular}{lcccc}
\hline & \multicolumn{2}{c}{ Amostras do ar } & \multicolumn{2}{c}{ Amostras de fezes } \\
\cline { 2 - 5 } & TOTAL & $\%$ & TOTAL & $\%$ \\
& $\mathbf{4 8}$ & $\mathbf{1 0 0}$ & $\mathbf{3 2}$ & $\mathbf{1 0 0}$ \\
\hline Amostras positivas para Cryptococcus sp & 1 & 2,1 & 9 & 28,1 \\
Amostras positivas para Cryptococcus & 0 & 0 & 1 & 3,1 \\
neoformans & & & & \\
\hline
\end{tabular}

\section{Discussão}

Cryptococcus neoformans e gattii são fungos patogênicos causadores de pneumonia e meningite em pacientes imunodebilitados e imunocompetentes. Essas espécies são encontradas no meio ambiente e adquiridas via inalação (QUINTERO, CASTAÑEDA, RUIZ, 2005; PUKKILA-WORLEY, MYLONAKIS, 2008;VELAGAPUDI et al., 2009).

Estudos sugerem uma correlação maior entre C. neoformans var. neoformans/ grubii e fezes de pombos e C. gattii e árvores. Entretanto, Grover e colaboradores (2009) sugerem que o habitat natural de ambas as espécies é mais amplo.

Neste estudo, 2,1\% das amostras do ar apresentaram Cryptococcus sp, entretanto $100 \%$ apresentaram ausência de crescimento para Cryptococcus neoformans. Já 3,1\% das amostras de fezes de pombos foram positivas para Cryptococcus neoformans e 29,1\%, para Cryptococcus sp. Esses dados relatam o primeiro caso de Cryptococcus neoformans isolado de amostras ambientais (fezes de pombos) na cidade de Votuporanga-São Paulo, visto que estudo anterior realizado por Rezende e colaboradores (2006) demonstrou ausência da levedura em amostras ambientais (fezes de pombos).

Hanasha e colaboradores (2004) analisaram 509 amostras de fezes de 
pombos coletadas de grandes cidades da Jordânia; dessas, 336 foram positivas para Cryptococcus sp; entretanto, não houve a presença de $C$. neoformans ou C. gattii.

Soogarun e colaboradores (2006) avaliaram 14 amostras de fezes de pombos obtidas de diferentes regiões de Bangkok - a presença de C. neoformans var. neoformans foi demonstrada em 9,09\% delas. Índice semelhante foi obtido em estudo na cidade do México, investigando amostras de fezes de aves, frutas e vegetais, com índices de positividades de 9,5\%,9,5\% e 4,2\% para Cryptococcus neoformans, respectivamente (LOPEZ-MARTINEZ, CASTANONOLIVARES, 1995).

Pal e colaboradores (1997) obtiveram $25 \%$ de fezes de pombos com $C$. neoformans em somente 28 amostras avaliadas. Índices elevados foram relatados por Granado e colaboradores (2005) em Bogotá, Colômbia.

No Brasil, análises ambientais têm relatado índices de isolamento maiores que o citado neste estudo. Filiú e colaboradores (2002) demonstraram a presença de C. nerformans var. grubii em 50\% das 20 amostras de excretas de aves de cativeiros na cidade de Campo Grande, Mato Grosso do Sul, Brasil. Dados semelhantes foram relatados por Abegg e colaboradores (2006), no Rio Grande do Sul, que isolaram C. neoformans var. grubii em $87 \%$ das amostras de excretas de psitáceos. Já Passoni e colaboradores (1999) encontraram em torno de $15 \%$ de positividade de isolamento de C. neoformans var. neoformans de excretas de aves em cativeiros, como canários, periquitos e outros psitacídeos, assim como na poeira doméstica na cidade do Rio de Janeiro.

Granados e Castañeda (2006) demonstrou que a prevalência de espécies e variedades de Cryptococcus varia entre cidades, sugerindo que condições climáticas e ambientais, tais como umidade, temperatura, radiação solar e evaporação, podem influenciar na presença desta levedura, sugerindo que o $C$. neoformans é mais frequentemente isolado nas estações mais úmidas e de fezes secas, sendo esta uma possível explicação para o resultado deste estudo. Entretanto, Quintero e colaboradores (2005) demonstraram presença de $C$. neoformans e $C$. gattii em amostras de fezes e de árvores, e uma maior incidência de isolamento em amostras coletadas no clima frio.

Vários estudos têm demonstrado que as excretas de pombos podem ser consideradas importantes substratos para a presença e manutenção da levedura na natureza (FILIÚ et al., 2002). A adaptação de pombos aos centros urbanos e às diferentes espécies de árvores constitui uma importante fonte ambiental de transmissão para a população, inclusive de poeira domiciliar (SWINNE et al., 
1989; PASSONI et al., 1998; PASSONI, 1999; CAMPISI et al., 2003; ERGIN et al., 2004). Estudo semelhante desenvolvido por Nishikawa e colaboradores (2003) analisou 80 amostras ambientais obtidas de excreta de pássaros, poeira doméstica, detritos de árvores e fezes de morcegos em diferentes regiões do Brasil, indicando diferenças regionais nas distribuições dos sorotipos e isolados de $C$. neoformans do ambiente urbano, não somente associados com excretas de pombos e aves, mas também às árvores.

A associação saprofítica do C. neoformans var neoformans/grubii com árvores, no Brasil, foi demonstrada por diferentes pesquisadores, sugerindo um possível habitat natural além das fezes de pombos (LAZERA et al., 1993; LAZERA et al., 1996; LAZERA et al., 2000). Em outros locais do mundo também foi relatada a associação de $C$. neoformans var grubii e árvores (BAUWENS et al., 1986; SWINNE, 1988; CAMPISI et al., 2003; GUGNANI et al., 2005; ERGIN et al., 2004).

\section{Conclusão}

A alta biodiversidade de C. neoformans, isolado de diversas fontes ambientais nas áreas urbanas do Brasil e em outros países, demonstrou a dificuldade de determinar o habitat natural dessa levedura. Estudos posteriores que estabeleçam as diversidades ambientais e sua manutenção no meio ambiente são necessários para uma compreensão da ecologia da levedura, fundamental na patogênese da criptococose, estabelecendo medidas de prevenção e controle ambiental na transmissão do patógeno.

\section{REFERÊNCIAS}

ABEGG, M.A.; CELLA, F.C.; FAGANELLO, J.; VALENTE, P.; SCHRANK, A.; VAINSTEIN, M.H. Cryptococcus neoformansi and Cryptococcus gattii isolated from the excreta of Psittaciformes in a Southern Brazilian Zoological Garden. Mycopathologia, New York, v.161, n.2, p.83-91, Feb 2006.

BALTAZAR, L.M.; RIBEIRO, M.A. Primeiro isolamento ambiental de Cryptococcus gattiii Estado do Espírito Santo. Rev Soc Brasil Med Trop, Uberaba, v.41, n.5, p.449-53, 2008.

BAUWENS, L.; SWINNE, D.; De VROEY, C.; De MEURICHY, W. Isolation of Cryptococcus neoformans var. neoformans in the aviaries of the Antwerp zoological gardens. Mikosen, v.29, n.7, p.291-294, Jul1986. 
CALLEJAS, A.; ORDOÑEZ, N.; RODROGUEZ, M.C.; CASTAÑEDA, E. First isolation of Cryptococcus neoformans var. gattii, serotype $C$, from the environment in Colômbia. Med Mycol., v.36, n.5, p.341-4, Oct 1998.

CAMPISI, E.; MANCIANTI, F.; PINI, G.; FAGGI, E.; GARGANI, G. Investigation in Central Italy of the possible association between Cryptococcus neoformans var. gattii and Eucalyptus camaldulensis. Eur J Epidemiol., v.18, n.4, p.357-62, 2003.

CASTAÑEDA, A.; MCEWN, J.; HIDALGO, M.; CASTAÑEDA, E. Cryptococcus spp DNA extraction from environmental samples. Biomédica, Spanish, v.24, n.3, p.324-31, Sep 2004.

DAVEL, G.; ABRANTES, R.; BRUDNY, M.; CÓRDOBA, S.; RODERO, L.; CANTEROS, C.E.; PERROTTA, D. 1st environmental isolation of Cryptococcus neoformans var. gattii in Argentina. Rev Argent Microbiol., Buenos Aires, v.35, n.2, p.110-2, Apr-Jun 2003.

ELLIS, D.; MARRIOTT, D.; HAJJEH, R.A.; WARNOCK, D.; MEYER, W.; BARTON, R. Epidemiology: surveillance of fungal infections. Med. Mycol., v.38, n.1, p.173-182, 2000.

ERGIN, C.; ILKIT, M.; HILMIOGLU, S.; KALELI, I.; GULBABA, A.G.; DEMIRCI, M.; KAYA, S. The first isolantion of Cryptococcus neoformans from Eucalyptus trees in South Aegean and Mediterranean Regions of Anatolia in Turkey despite Taurus Mountains alkalinity. Mycopathologia, New York, v.158, n.1, p.43-7, Jul 2004.

ESCANDÓN, P.; QUINTERO, E.; GRANADOS, D.; HUÉRFANO, S.; RUIZ, A.; CASTAÑEDA, E. Isolation of Cryptococcus gattii serotype B from detritus of Eucalyptus tree in Colômbia. Biomédica, Spanish, v.25, n.3, p.390-7, Sep. 2005.

FILIÚ, W.F.O.; WANKE, B.; AGUENA, S.M.; VILELA, V.O.; MACEDO, R.C.L.; LAZERA, M. Cativeiro de aves como fonte de Cryptococcus neoformans na cidade de Campo Grande. Revista da Sociedade Brasileira de Medicina Tropical, v.35, n.6, p.591-595, Nov-Dec 2002.

FORTES, S.T.; LAZERA, M.S.; NISHIKAWA, M.M.; MACEDO, R.C.; 
WANKE, B. First isolation of Cryptococcus neoformans var. gattii from a native jungle tree in the Brazilian Amazon rainforest. Mycoses, v.44, n.5, p.137-40, 2001.

GRANADOS, D.P.; CASTAÑEDA, E. Influence of climatic conditions on the isolation of members of the Crytpococcus neoformans species complex from trees in Colombia from 1992-2004. FEMS Yeast Res, v.6, n.4, p.636-44, Jun 2006.

GROVER, N.; NAWANGE, S.R.; NAIDU, J.; SINGH, S.M.; SHARMA, A. Ecological niche of Cryptococcus neoformans var. grubii and Cryptococcus gattii in dcaying wood of trunk hollows of living trees in Japalpur City of Central Índia. Mycopathologia, New York, v.168, n.1, p. 49-50, Oct. 2009.

GUGNANI, H.C.; MITCHELL, T.G.; LITVINTSEVA, A.P.; LENGELER, K.B.; HEITMAN, J.; KUMAR, A.; BASU, S.; PALIWAL-JOSHI, A. Isolation of Cryptococcus gattii and Cryptococcus neoformans var. grubii from the flowers and bark of Eucalyptus trees in Índia. Med Mycol., v.43, n.6, p.565-9, Sep 2005.

HAMASHA, A.M.; VILDIRAN, S.T.; GONLUM, A.; SARACLI, M.A.; DOGANCI, L. Cryptococcus neoformans varietis from material under the canopies of eucalyptus trees and pigeon dropping samples from four major cities in Jordan. Mycopathologia, New York, v.158, n.2, p.195-9, Aug 2004.

KROCKENHERGER, M.B.; CANFIELD, P.J.; MALIK, R. Cryptococcus neoformans in the koala: colonization by $\mathrm{C} n$ var gattii and investigation of environmental sources. Med Mycol., v.40, n.3, p.263-72, Jun 2002.

LAZÉRA, M.S.; CAVALCANTI, M.A.S.; LONDERO, A.T.; TRILLES, L.; NISHIKAWA, M.M.; WANKE, B. Possible primary ecological niche of Cryptococcus neoformans. Medical Mycology, v.38, n.5, p.379-383, 2000.

LAZÉRA, M.S.; PIRES, F.D.A.; CAMILO-COURA, L.; NISHIKAWA, M.M.; BEZERRA, C.C.F.; TRILLES, L. et al. Natural habitat of Cryptococcus neoformans var. neoformans in decaying wood forming hollows in living trees. Journal of Medical and Veterinary Mycology, v.34, n.2, p.127-131, MarApr 1996. 
LAZÉRA, M.S.; WANKE, B.; NISHIKAWA, M.M. Isolation of both varieties of Cryptococcus neoformans from saprophytic sources in the city of Rio de Janeiro, Brazil. Journal of Medical and Veterinary Mycology, v.3, p.449-454, 1993.

LOPEZ-MARTINEZ, R.; CASTANON-OLIVARES, L.R. Isolation of Cryptococcus neoformans var. neoformans from bird droppings, fruits and vegetables in Mexico City. Mycopathologia, New York, v.129, n.1, p:2528, 1995.

NISHIKAWA, M.M.; LAZERA, M.S.; BARBOSA, G.G.; TRILLES, L.; BALASSIANO, B.R.; MACEDO, R.C.L. et al. Serotyping of 467

Cryptococcus neoformansi isolates from Clinical and environmental sources in Brazil: analysis of host and regional patterns. Journal of Clinical Microbiology, v.41, n.1, p.73-77, Jan 2003.

PAL, M. First report of isolation of Cryptococcus neoformans var neoformans from avian excreta in Kathmandu, Nepal. Rev Iberoam Micol., v.14, n.4, p.181-3, Dec 1997.

PASSONI, L.F.C. Wood, animals, and human beings as reservoir for human Cryptococcus neoformans infection. Revista Iberoamericana de Micologia, v.16, p.77-81, 1999.

PASSONI, L.F.; WANKE, B.; NISHIKAWA, M.M.; LAZERA, M.S. Cryptococcus neoformans isolated from human dwellings in Rio de Janeiro, Brazil: an analysis of the domestic enrironment of AIDS patients with and without cryptococcosis. Med Mycol., v.36, n.5, p.305-11, Oct 1998.

PASSONI, L.F.C.; WANKE, B.; NISHIKAWA, M.M.; LAZERA, M.S. Cryptococcus neoformans isolated from human dwellings in Rio de Janeiro, Brazil: An analysis of domestic environment of AIDS patients with and without cryptococcosis. Medical Mycology, v.36, n.5, p.305-11, Oct 1998.

PUKKILA-WORLEY, R.; MYLONAKIS, E. Epidemiology and management of cryptococcal meningitis: developments and challenges. Expert Opin Pharmacother, v.9, n.4, p.551-60, Mar 2008.

QUINTERO, E.; CASTAÑEDA, E.; RUIZ, A. Environmental distribution of 
Cryptococcus neoformans in the department of Cundinamarca-Colombia. Rev. Iberoam Micol., v.22, n.2, p93-8, Jun 2005.

REZENDE, C.; MUNHÓZ, C.J.M.; ALMEIDA, G.G. Investigação Ambiental de Cryptococcus neoformans na Cidade de Votuporanga - São Paulo.

Newslab, v.87, p.88-94, 2008.

SOOGARUN, S.; WIWANITKIT, V.; PALASUWAN, A.; PRADNIWAT, P.; SUWANSAKSRI, J.; LERTLUM, T.; MAUNGKOTE, T. Detection of Cryptococcus neoformans in BIRD excreta. Southeast Asian J Trop Med Public Health, v.37, n.4, p.768-70, Jul. 2006.

SWINNE, D.; DEPPNER, M.; LAROCHE, R.; FLOCH, J.J.; KADENDE, P. Isolation of Cryptococcus neoformans from houses of AIDS-associated cryptococcosis patients in Bujumbura (Burundi). AIDS, v.3, n.6, p.389-390, Jun 1989.

SWINNE D. Ecology of Cryptococcus neoformans and epidemiology of cryptococcosis in the old World. In: Torres-Rodriguês, J. M., ed. In: CONGRESS OF THE INTERNATIONAL SOCIETY FOR HUMANAND ANIMAL MYCOLOGY, 10., Proceedings... Barcelona: Prous Scientific, 1988

VELAGAPUDI, R.; HSUEH, Y.P.; GEUNES-BOYER, S.; WRIGHT, J.R.; HEITMAN, J. Spores as infectious propagules of Cryptococcus neoformans. Infect Immun, v.77, n.10, p.4345-55, Jul. 2009.

Title: Cryptococcus Neoformans: the First Reported Case of Isolation in Pigeon Excreta in the City of Votuporanga-São Paulo

\section{ABSTRACT}

Cryptococcocus neoformans and Cryptococcus gattii are closely related pathogenic fungi that cause pneumonia and meningitis in both immunocompromised and immunocompetent hosts. Both species are found in the environment, and are acquired via inhalation, leading to an initial pulmonary infection. We evaluated 48 air samples and 32 samples of pigeon excreta from June to August, 2007. The presence of capsule was examined with Indian ink preparation. They were also tested for urease and phenoloxidase enzymes. Cryptococcus neoformans was recovered from pigeon excreta in 
3.1\%. The results suggest that climatic conditions can affect the occurrence of the yeast in different environmental sources.

KEYWORDS: Excreta; Air; Cryptococcus neoformans; Votuporanga. 